International Egyptian Journal of Nursing Sciences and Research

(IEJNSR)

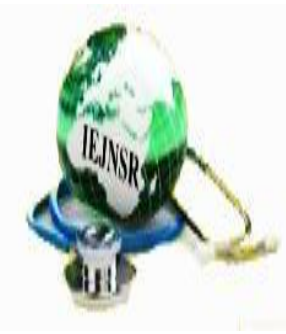

Received 2020-11-28

Review article

Accepted 2020-12-2

Published2021-1-4

\title{
Novel coronavirus disease (COVID-19) in pregnancy
}

Asmaa Anwar Abd elglil

Women's Health and Maternity Nursing, Faculty of Nursing Kafrelsheikh

University, Kafrelsheikh, Egypt

asmanour75@yahoo.com

\begin{abstract}
Pregnancy is a condition in which the immune system is at risk of viral infections, and the morbidity is increased within seasonal influenza. Therefore, the COVID-19 epidemic may additionally have serious penalties for pregnant women. There seems to be some hazard of untimely rupture of membranes, preterm delivery, fetal tachycardia, and fetal misery when the contamination happens in the $1 / 3$ trimester of pregnancy. Clinical tips for managing COVID-19 contamination in being pregnant have to be primarily based on records from the contemporary epidemic as a substitute than drawing on a restricted journey from preceding outbreaks of unique sorts of coronaviruses, as their epidemiology, medical route, and response to cure may also differ. Guidelines will evolve as extra records end up accessible and the journey is gathered. Therefore, whole records on all pregnancies affected through COVID19 ought to be accumulated and made publicly available. Sharing data, expertise and assisting international locations with terrible sources and weaker healthcare structures are essential in this respect.
\end{abstract}

Keywords: Covid-19, Corona Virus, Pregnancy

\section{Introduction}

Pregnant females or lately pregnant girls who are older, overweight, and have existing clinical stipulations such as hypertension and diabetes appear to have an accelerated danger of growing extreme COVID-19. When pregnant girls increase extreme disease, they additionally appear to greater frequently require care in intensive care gadgets than the non-pregnant female of reproductive age. Due to modifications in their bodies and immune systems, we are aware that pregnant girls can be badly affected by some respiratory infections. It is consequently necessary that they take precautions to shield themselves towards COVID-19, and file viable signs (including fever, cough, or challenge in breathing) to their healthcare provider (1). 


\section{Diagnosis}

The essential medical manifestatio ns are increased body temperature, tiredness, myalgia, dry cough, and shortness of breath. Few sufferers may additionally exist with nasal congestion, runny nose, sore throat, hemoptysis, or diarrhea. A computed tomography (CT) scan of the chest besides distinction is the most beneficial investigation to affirm or rule out viral pneumonia, and ought to be carried out in suspected instances as the chance of radiation publicity to the fetus is very small. In a current report, the sensitivity of chest CT in diagnosing COVID-19 was once proven to be increased than that of RT-PCR $(98 \%$ vs $71 \%$ ). Radiological symptoms of viral pneumonia have been existing in an overwhelming majority of stated pregnancies with COVID-19 infection.SARS-COV-2 is the etiologic agent of COVID-19, and its viral nucleic acid detection the use of real-time polymerase chain response (RT-PCR) is viewed as the reference widespread for the diagnosis. Specimens ought to be bought from saliva, higher respiratory tract (nasopharyngeal and oro-pharyngeal swabs), lower respiratory tract urine, and stool if possible (2).

\section{Symptoms and disease severity}

Similar to non-pregnant patients, the fundamental competencies of COVID-19 in being pregnant are increased body temperature, cough, dyspnea, and lymphopenia and are similarly introduced in Table. Shortness of breath is described in up to $18 \%$ of sufferers with COVID-19(3).

\section{High-risk groups}

while, some researchers have raised worries that pregnant girls may additionally be greater volatile for COVID-19 as, in general, they may additionally be greater inclined to respiratory contamination the proof to date would advise that they are no greater susceptible to this specific pathogen

than the universal populace (4).

\section{Matrnal mortality}

Initial lookup proposes a -long way reduce the rate of maternal mortality for these with proven COVID-19

in evaluation to the ones infected with MERS and SARS, respectively. A systematic view of forty-one pregnancies uncovered to COVID19 located maternal mortality of $0 \%$ in contrast to a rate of $28.6 \%$ with MERS and $25.8 \%$ with SARS, whilst the 2d file of 108 pregnancies additionally suggested no maternal deaths. (5)

\section{Caesarian sections rate}

The

cesarean section (CS) charge for girls wit $\mathrm{h} \quad$ COVID-19 contamination has been stated as ranging from $42.9 \%$ to as excessive as $91-$ 92\% in different studies(6).

\section{Preterm Delivery and Neonatal morbidity}

Preliminary

opinions stated immoderate costs of preterm labor, ranging from forty-one to forty-seven percent.

systematic assessment of

33 research because of this described the consequences of 385 pregnant girls with COVID-19 with gestational age at delivery ranging from 30 to 41 weeks' gestation and preterm delivery of $15.2 \%$ (7). While many of the preterm deliveries had been iatrogenic and for maternal reasons, there are reviews of fetal distress as the indication in some cases, even though in others the indication for transport is unclear (8). At present, there is inadequate proof to decide any correlation between spontaneous preterm labor and COVID19 contamination in being pregnant even though there are some pronounced instances of preterm prelabour rupture of membranes. (10)

\section{Prevention}


The incubation duration of COVID19 is about 2-14 days, however, contaminated individuals can transmit the virus through shut contact and respiratory droplets possibly even earlier than they turn out to be symptomatic. Physiological modifications in the immune and respiratory machine can also make pregnant girls greater inclined to COVID-19 infection in the course of the epidemic. No wonderful vaccine is on hand at present. Therefore, it is really helpful that

pregnant female chorus from needless tra vel, keep away from crowds, public transport, contact with unwell people, and extra importantly, exercise and keep suitable non-public and social hygiene. Pregnant ladies with signs of fever, cough, fatigue, myalgia, sore throat, or shortness of breath need to are looking for a well-timed clinical session and help. Women with journey records to endemic areas and these with a scientific suspicion of contamination must be remote and investigated. Some pregnant females may additionally increase extreme anxiousness and despair requiring expert psychological aid to stop destructive consequences (11).

\section{Treatments}

The therapy of a suspected COVID-19 affected person starts at presentation to the place of the affected person ought to be met by using healthcare personnel in suitable PP $E$ at a targeted entrance, separate from that of hobbies antenatal care. The affected person ought to be furnished with masks and delivered to an isolation room. The affected person ought to be assessed rapidly, and a selection made concerning discharge to the neighborhood or admission to a health center isolation room. The preliminary evaluation ought to encompass assessing the severity of symptoms, inclusive of chronic pyrexia, progressing shortness of breath, hemoptysis, and chest pain, as properly as

the administration of different clinical co morbidities as outlined above (11). A multi-disciplinary crew strategy ought to be essential to the remedy of the pregnant affected person with extreme COVID-19 and have to consist of Obstetrics, Infectious Diseases/Respiratory team, Microbiology, Midwifery and Pharmacology (12).

\section{Thromboprophylaxis}

There are rising reviews of a multiplied incidence of each venous and arterial thromboembolism in sufferers recognized with COVID-19 due to immoderate inflammation, hypoxia, immobilization, and diffuse intravascular coagulation. (13)

\section{Corticosteroid}

No proof giving steroids for fetal lung maturation motives damage in the context of COVID-19 infection. There are reviews of worse effects for sufferers with the use of corticosteroids; however, the traditional corticosteroids used in the pregnant female are roughly one-fourth to one-tenth of the quantity used in these publications(14).

\section{Antenatal care}

It is endorsed that all pregnant females take a look at social distancing and observe selfisolation coaching to forestall publicity to COVID-19 and exercise correct hand hygiene (15). FIGO currently recommends that for the duration of the direction of the pandemic, the familiar precept has to be to decrease inperson workplace visits and if realistic and appropriate, reflect on consideration on appointments by using smartphone or videoconferencing. Women with signs of COVID-19 need to be examined and appointments delayed if possible, in the course of the length of self-quarantine. If signs and symptoms persist, they ought to name and make an appointment 
for checking out and/or hospitalization.

Maternity gadgets ought to think

about extra measures,

and

these must encompass limiting

the variety of aid persons/visitors

with sufferers for outpatient and inpatient

visits, along

with labor

and transport areas (16).

\section{Labor and delivery}

For the female who is both suspected and verified COVID-19 positive, splendid care need to be taken at the time of labor and delivery. As referred to above neither CS nor vaginal shipping confers

any extra dangers to both the fetus and his mother, and mode of transport ought to be decided on an individualized basis. Adherences to contamination precautions are essential and ought to be deliberate in development at a nearby level. This is especially essential in lowincome international locations with confined resources. Staff attending to these females have to be supplied with the essential non-public protecting tools (PPE) (17). At present, there is no research recommending fetal monitoring of asymptomatic pregnant women. Continuous CTG monitoring must be supplied to symptomatic sufferers in labor (18).

\section{Breastfeeding}

Concerning breastfeeding, in a female who is COVID-19 positive, the most important hazard for kiddies is the shut contact with the mother, who is probably to shed infective airborne droplets. The lookup from China, though limited, has no longer proven a virus in breast milk, and in mild of the cuttingedge evidence, the advantages of breastfeeding appear to outweigh any achievable dangers of the transmission of the virus thru breast milk. (19)

\section{References}

1. World Health Organization. Coronavirus disease (COVID-19) pandemic. Available at:https://www.who.int/emergencies/ diseases/novelcoronavirus- 2019.

Accessed June 17, 2020.

2. Wu YC, Chen CS, Chan YJ. The outbreak of COVID-19: an overview. J Chin Med Assoc 2020;83:217-20.

3. Di Mascio D, Khalil A, Saccone G, et al. Outcome of coronavirus spectrum infections (SARS, MERS, COVID-19) during pregnancy: a systematic review and meta-analysis. Am J Obstet GynecolMFM2020 [Epub ahead of print].

4. Royal College of Obstetricians \& Gynaecologists. Coronavirus (COVID-19) infection in pregnancy. Information for healthcare professionals. Version 8. 2020. Available at: https://www.rcog.org.uk/globalassets /documents/guidelines/2020-04-17coronavirus-covid-19- infection-inpregnancy.pdf. Accessed May 11,

5. National Health Commission of China. New coronavirus pneumonia prevention and control program [in Chinese]. 4th ed. 2020. Available at: http://www.gov.cn/zhengce/zhengce $\mathrm{ku} / 2020-$

01/28/5472673/files/0f96c10cc09d4 d36a6f9a f0b42d972b.pdf. Accessed March 14, 2020.

6. Chen H, Guo J, Wang C, et al. Clinical characteristics and intrauterine vertical transmission potential of COVID-19 infection in nine pregnant women: a retrospective review of medical records. Lancet 2020;395:809-15.

7. Chen S, Huang B, Luo DJ, et al. Pregnant women with new coronavirus infection: a clinical characteristics and placental pathological analysis of three cases. Zhonghua Bing Li Xue Za Zhi 2020;49:E005.

8. Moher D, Liberati A, Tetzlaff J, et al, and the PRISMA Group. Preferred Reporting Items for Systematic Reviews and Meta- 
Analyses: The PRISMA Statement. Ann Intern Med 2009;151: 264-9.

9. Mullins E, Evans D, Viner RM, O'Brien P, Morris E. Coronavirus in pregnancy and delivery: rapid review. Ultrasound Obstet Gynecol [In

10. press].

11. Murad MH, Sultan S, Haffar S, Bazerbachi F. Methodological quality and synthesis of case series and case reports. BMJ Evid Based Med 2018;23:60-3.

12. Yang H, Wang C, Poon LC. Novel coronavirus infection and pregnancy. Ultrasound Obstet Gynecol 2020;55:435-7.

13. Zhang L, Jiang $Y$, Wei $M$, et al. Analysis of the pregnancy outcomes in pregnant women with COVID-19 in Hubei Province.

14. Zhonghua $\mathrm{Fu}$ Chan $\mathrm{Ke} \mathrm{Za} \mathrm{Zhi}$ 2020;55: E009.

15. Zhu H, Wang $\mathrm{L}$, Fang $\mathrm{C}$, et al. Clinical analysis of 10 neonates born to mothers with 2019-nCoV pneumonia. Transl Pediatr 2020;9: 51-60.

16. Schuchat A. Reflections on pandemics, past and present. Am J Obstet Gynecol 2011;204(6 Suppl 1):S4-6.

17. Adhikari EH, Nelson DB, Johnson $\mathrm{KA}$, et al. Infant outcomes among women with Zika virus infection during pregnancy: results of a large prenatal Zika screening program. Am J Obstet Gynecol 2017;216: 292.e1-8.

18. Avram CM, Greiner KS, Tilden E, Caughey AB. Point-of-care HIV viral load in pregnant women without prenatal care: a costeffectiveness analysis. Am J Obstet Gynecol 2019;221:265.e1-9. 